\title{
Solidification in a Supercomputer: From Crystal Nuclei to Dendrite Assemblages
}

\author{
YASUSHI SHIBUTA, ${ }^{1,4}$ MUNEKAZU OHNO ${ }^{2}$ and TOMOHIRO TAKAKI ${ }^{3}$ \\ 1.-Department of Materials Engineering, The University of Tokyo, 7-3-1 Hongo, Bunkyo-ku, \\ Tokyo 113-8656, Japan. 2.-Division of Materials Science and Engineering, Faculty of Engi- \\ neering, Hokkaido University, Kita 13 Nishi 8, Kita-ku, Sapporo, Hokkaido 060-8628, Japan. \\ 3.-Faculty of Mechanical Engineering, Kyoto Institute of Technology, Matsugasaki, Sakyo-ku, \\ Kyoto 606-8585, Japan. 4.—e-mail: shibuta@material.t.u-tokyo.ac.jp
}

Thanks to the recent progress in high-performance computational environments, the range of applications of computational metallurgy is expanding rapidly. In this paper, cutting-edge simulations of solidification from atomic to microstructural levels performed on a graphics processing unit (GPU) architecture are introduced with a brief introduction to advances in computational studies on solidification. In particular, million-atom molecular dynamics simulations captured the spontaneous evolution of anisotropy in a solid nucleus in an undercooled melt and homogeneous nucleation without any inducing factor, which is followed by grain growth. At the microstructural level, the quantitative phase-field model has been gaining importance as a powerful tool for predicting solidification microstructures. In this paper, the convergence behavior of simulation results obtained with this model is discussed, in detail. Such convergence ensures the reliability of results of phase-field simulations. Using the quantitative phase-field model, the competitive growth of dendrite assemblages during the directional solidification of a binary alloy bicrystal at the millimeter scale is examined by performing two- and threedimensional large-scale simulations by multi-GPU computation on the supercomputer, TSUBAME2.5. This cutting-edge approach using a GPU supercomputer is opening a new phase in computational metallurgy.

\section{INTRODUCTION}

Many practical metals and alloys undergo solidification during their production. ${ }^{1,2}$ Since their microstructure directly affects the properties of products, it is essential to control the microstructure of metals and alloys during the solidification with a high degree of accuracy. Despite considerable effort over many years, it is still challenging to control the solidification microstructure as planned. This is mainly due to the following three reasons:

(1) The difficulty of direct (in situ) observation.

(2) The wide range of temporal and spatial scales.

(3) The need to consider multiple physics including fluid flow, thermal and solute diffusion.

Regarding the first difficulty, several pioneering works have achieved the in situ observation of solidification for transparent materials ${ }^{3,4}$ and for alloys by using synchrotron radiation x-rays. ${ }^{5-7}$ These studies provided considerable information on dendrite growth. However, it is not yet straightforward to directly observe the dynamics of solidification during actual production processes in general. Therefore, computational studies have contributed to clarifying the nature of solidification processes. In the early stage of research, Monte Carlo simulations with the Pott model ${ }^{8}$ were often performed to study the kinetics of grain growth, ${ }^{9,10}$ and this became a popular method for the study of solidification, recrystallization and other phenomena. ${ }^{11,12}$ The front tracking method ${ }^{13,14}$ and cellular automata ${ }^{15,16}$ have also been widely employed for simulations of dendritic growth. In 1993, Kobayashi succeeded in reproducing a complicated dendrite structure using the phase-field model. ${ }^{17}$ Since then, phase-field simulation $^{18-23}$ has become a major tool for the simulation of solidification. The main advantage of 
the phase-field model is that it is not necessary to explicitly track the position of a sharp interface in complex microstructural patterns. On the other hand, a long-standing issue regarding a quantitative aspect of the phase-field model remained unresolved until recently. This serious problem has been resolved by recent progress in the quantitative phase-field model. ${ }^{24-33}$ Combined with the recent rapid progress in high-performance computational environments, large-scale phase-field simulations can now capture the competition between bundles of dendrites, including selection and regularity, ${ }^{34-36}$ which is filling the gaps in knowledge over a wide range of temporal and spatial scales. Moreover, the coupling of phase-field simulation with computational fluid dynamics is now capturing some aspects of multiple physics during solidification, ${ }^{37,38}$ which can be applied to fluid-dynamics-based phenomena such as the fragmentation of dendrite tips during solidification. ${ }^{38}$ The Lattice Boltzmann method $^{39,40}$ is a promising numerical method for a large-scale fluid computation in solidification problem.

In combination with phase-field simulations, molecular dynamics simulations have contributed to the estimation of interfacial parameters such as the solid-liquid interfacial energy and the kinetic coefficient. ${ }^{41-49}$ In particular, the anisotropy in the interfacial parameters is a key factor determining the morphology of dendrite structures, although there are few reliable experimental values for the degree of anisotropy in the interfacial parameters. Therefore, an important role of molecular dynamics simulations is to provide interfacial parameters estimated from atomic-scale information for use in mesoscale simulations, which is a basic concept of multiscale modeling. Moreover, recent large-scale molecular dynamics simulations have captured the morphological dynamics of crystal growth with curved interfaces ${ }^{50-52}$ and multiple nucleation from an undercooled melt, which is followed by the formation of polycrystalline microstructures. ${ }^{53,54}$

Such progress in simulations of solidification is largely attributable to the rapid progress in highperformance computational environments. In particular, considerable benefit has been obtained from the high parallel efficiency of graphics processing units (GPUs). Large-scale simulations performed on GPU supercomputers have ranged from the nucleation and subsequent grain growth in a millionatom molecular dynamics simulation to the competitive growth of millimeter-size dendrite assemblages in a large-scale phase-field simulation. In this paper, cutting-edge simulations of solidification performed on a GPU supercomputer are introduced with a brief introduction to the current state of computational studies on solidification. Firstly, the spontaneous evolution of anisotropy in a solid nucleus investigated by million-atom molecular dynamics simulation is discussed in "Solidification in Large-Scale Molecular Dynamics Simulation" section. Investigation of the nucleation of crystal nuclei from an undercooled melt, which is an initial stage of solidification, is also outlined in the same section. In "Advances in Quantitative Computation of Solidification Microstructures" section, advances in quantitative computation of phase-field simulations are discussed with an examination of the convergence of the results of quantitative phasefield simulations. In "Large-Scale Phase-Field Simulation of Competitive Growth of Dendrite Assemblages" section, we report the competitive growth of dendrite assemblages during the directional solidification of a binary alloy bicrystal investigated by performing two- and threedimensional large-scale simulations using the quantitative phase-field model by multi-GPU computation. We conclude with a discussion of the implications of these results for the future of computational metallurgy.

\section{SOLIDIFICATION IN LARGE-SCALE MOLECULAR DYNAMICS SIMULATION}

\section{Spontaneous Evolution of Anisotropy in a So- lid Nucleus}

As described above, molecular dynamics simulations have contributed to the estimation of interfacial properties. The estimated values of these properties in representative papers ${ }^{41-49}$ are summarized in Table I. As can be seen in the table, the kinetic coefficient of the bcc $\langle 100\rangle$ orientation is slightly higher than those of the $\langle 110\rangle$ and $\langle 100\rangle$ orientations in general. Such a difference in the kinetic coefficient as well as that in the interfacial energy causes anisotropy in the solid nucleus, which results in dendritic growth in accordance with the interfacial stability. Therefore, it is reasonable for an anisotropic morphology to be generated during solidification in a phase-field simulation when the effect of anisotropy is taken into account in the interfacial parameters. In turn, it should be possible in principle to achieve an anisotropic morphology even in a molecular dynamics simulation if the system size is sufficiently large. Then, what is the critical size for obtaining clear anisotropy in a solid nucleus in an atomic-scale simulation? At least we did not observe such anisotropy in a solid nucleus during solidification in a cubic cell of side $15 \mathrm{~nm} .{ }^{46,55}$ Therefore, a much larger system should be employed to discuss this issue, which is, however, computationally demanding.

We have developed our own code for carrying out large-scale molecular dynamics simulations by single-GPU computing, which enables molecular dynamics simulations of 1 million atoms to be handled over a period of nanoseconds with a computational time of several days. ${ }^{56}$ Using this code with singleGPU computing, the spontaneous evolution of anisotropy in a solid nucleus during the solidification of iron was investigated by million-atom molecular dynamics simulation. ${ }^{52}$ As the simulation methodology, the Finnis-Sinclair potential, ${ }^{57}$ which is one 
Table I. Thermodynamic and kinetic parameters estimated from molecular dynamics simulations ${ }^{41-49}$

\begin{tabular}{|c|c|c|c|c|c|c|}
\hline Element (potential) & $\boldsymbol{T}_{\mathbf{M}}(\mathbf{K})$ & $\Delta H($ eV/atom $)$ & $\sigma_{\mathrm{SL}}\left(\mathbf{m} \mathbf{J} / \mathbf{m}^{2}\right)$ & $\mu_{\langle\mathbf{1 0 0}\rangle}(\mathbf{c m} / \mathbf{s K})$ & $\mu_{\langle\mathbf{1 1 0}\rangle}(\mathbf{c m} / \mathbf{s K})$ & $\mu_{\langle 111\rangle}(\mathbf{c m} / \mathbf{s K})$ \\
\hline $\mathrm{Fe}(\mathrm{bcc})(\mathrm{FS})^{\mathrm{a}}$ & $2400 \pm 10[1811.0]$ & $0.22[0.143]$ & 170 & 30.5 & 25.7 & - \\
\hline $\mathrm{Fe}(\mathrm{bcc})(\mathrm{ABCH})^{\mathrm{b}}$ & $2358.7 \pm 4.0$ & 0.218 & $206 \pm 10$ & $32.5 \pm 1.6$ & $24.6 \pm 1.4$ & $25.6 \pm 2.0$ \\
\hline $\mathrm{Fe}(\mathrm{bcc})(\mathrm{MH}(\mathrm{SA}))^{\mathrm{b}}$ & $1772.0 \pm 2.0$ & 0.162 & $175 \pm 11$ & $45.8 \pm 3.8$ & $33.5 \pm 1.0$ & $31.8 \pm 1.8$ \\
\hline $\mathrm{Fe}(\mathrm{bcc})(\text { pair })^{\mathrm{b}}$ & $2311.8 \pm 3.0$ & 0.259 & $221 \pm 14$ & $30.8 \pm 7.0$ & $22.6 \pm 4.0$ & $23.1 \pm 3.6$ \\
\hline $\mathrm{Fe}(\mathrm{bcc})(\mathrm{MEAM})^{\mathrm{c}}$ & 1807 & 0.134 & $184 \pm 14$ & - & - & - \\
\hline $\mathrm{Fe}(\mathrm{fcc})(\mathrm{ABCH})^{\mathrm{b}}$ & $2251.0 \pm 6.0$ & 0.200 & $319 \pm 12$ & $24.0 \pm 1.6$ & $19.3 \pm 2.2$ & - \\
\hline $\mathrm{Fe}($ fcc $)(\text { pair })^{\mathrm{b}}$ & $2202.0 \pm 7.0$ & 0.212 & $311 \pm 14$ & $\begin{array}{c}2+.01 .0 \\
-\end{array}$ & $\begin{array}{c}10.0+2.4 \\
-\end{array}$ & - \\
\hline $\mathrm{Ni}(\mathrm{SMF})^{\mathrm{d}}$ & $1820[1726]$ & $0.187[0.1776]$ & $310 \pm 7$ & - & - & - \\
\hline $\mathrm{Ni}(\mathrm{FBD})^{\mathrm{e}, \mathrm{f}}$ & 1714 & 0.179 & $284.7 \pm 7.0$ & 45 & 32 & 18 \\
\hline $\mathrm{Ni}(\mathrm{SC})^{\mathrm{g}}$ & 1205 & 0.11 & 256 & - & - & - \\
\hline $\mathrm{Ni}(\mathrm{MEAM})^{\mathrm{h}}$ & 1741.6 & 0.23 & 331.8 & & & \\
\hline $\mathrm{Cu}(\mathrm{FBD})^{\mathrm{e}, \mathrm{f}}$ & $1279[1356.6]$ & $0.116[0.135]$ & $195.7 \pm 5.6$ & 46 & 27 & 19 \\
\hline $\mathrm{Cu}(\mathrm{MEAM})^{\mathrm{h}}$ & 1319.6 & 0.15 & 255.0 & - & - & - \\
\hline $\mathrm{Al}(\mathrm{EA})^{\mathrm{i}}$ & 930 [933.5] & $0.101[0.109]$ & 149 & - & - & - \\
\hline $\mathrm{Al}(\mathrm{MEAM})^{\mathrm{h}}$ & 924.9 & 0.12 & 172.6 & & & \\
\hline $\mathrm{Cr}(\mathrm{SC})^{\mathrm{g}}$ & 2232 [2180] & $0.16[0.22]$ & 304 & - & - & - \\
\hline
\end{tabular}

$T_{\mathrm{M}}, \Delta H, \sigma_{\mathrm{SL}}$ and $\mu$ represent the melting point, latent heat, solid-liquid interfacial energy and kinetic coefficient (the subscript shows the orientation), respectively. Experimental values are listed in parentheses ${ }^{\mathrm{a}}$ Watanabe et al. ${ }^{46 \mathrm{~b}}$ Sun et al. ${ }^{45 \mathrm{c}}$ Asadi et al. ${ }^{48 \mathrm{~d}} \mathrm{Asta}$ et al. ${ }^{42 \mathrm{e}} \mathrm{Hoyt}$ et al. ${ }^{41 f}$ Hoyt et al. ${ }^{44 g}$ Hashimoto et al. ${ }^{47 \mathrm{~h}}$ Asadi et al. ${ }^{49 \mathrm{i}}$ Morris ${ }^{43}$.

of the established potentials for bcc metals, was employed for the interaction between iron atoms. A leapfrog method was used to integrate a classical equation of motion with a time step of $5.0 \mathrm{fs}$. A Berendsen thermostat ${ }^{58}$ was applied to control the temperature. The Andersen method ${ }^{59}$ was employed to control the pressure in each direction independently. The initial configuration was prepared by embedding an octagonal solid nucleus in an iron melt. The iron melt was obtained in advance by heating a bcc crystal of iron of size $53.4 \times 53.4 \times 4.3 \mathrm{~nm}^{3}(1,037,880$ atoms $)$ at $3500 \mathrm{~K}$ under a NVT constant condition. The solid nucleus was prepared as an octagonal cutout from the bcc crystal with four $\{100\}$ facets and four $\{110\}$ facets of side $1.78 \mathrm{~nm}$. The solid nucleus was inserted into the center of the iron melt, while omitting all melt atoms located within $2.5 \AA$ from solid atoms. After the quenching of the simulation cell at $10 \mathrm{~K}$ for $25 \mathrm{ps}$ to fill the gap between the melt and solid atoms, the obtained initial configuration was isothermally undercooled at $\Delta T=300 \mathrm{~K}$. Note that the melting point of bcc Fe according to the FinnisSinclair potential is $2400 \mathrm{~K},{ }^{60}$ and therefore the undercooling temperature of $300 \mathrm{~K}$ corresponds to $2100 \mathrm{~K}$.

Figure 1 shows snapshots of the atomic configuration during the spontaneous evolution of anisotropy in the solid nucleus during solidification. ${ }^{52}$ Although the edges of the octagonal nucleus are smoothed and the nucleus takes a spherical shape in the initial stage, the spherical nucleus gradually preferentially grows in the $\langle 100\rangle$ direction from approximately $300 \mathrm{ps}$. Then, it grows to form a rhombic-like structure with fourfold symmetry at $500 \mathrm{ps}$. The shape of the solid-liquid interface in the

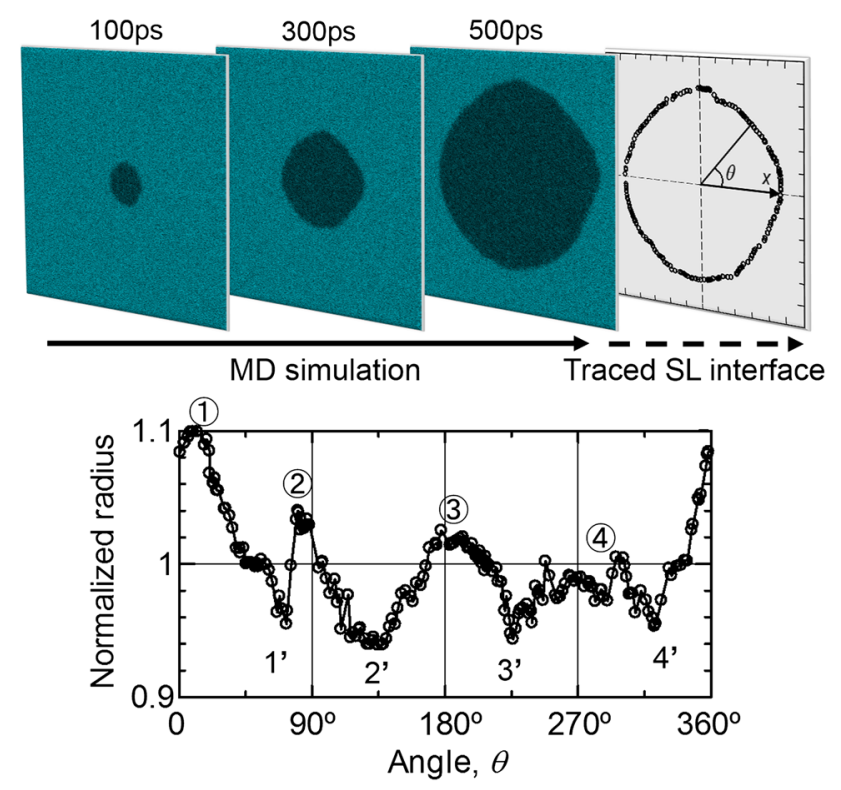

Fig. 1. Million-atom molecular dynamics simulation of spontaneous evolution of anisotropy in solid nucleus during solidification. ${ }^{52} \mathrm{Re}-$ sults from Ref. 52 are reconstructed. (Top) Snapshots at 100, 300 and $500 \mathrm{ps}$ and the trace of the solid-liquid interface obtained from the snapshot at 500 ps. (Bottom) Normalized radius of the solid nucleus as a function of rotation angle $\theta$.

snapshot at $500 \mathrm{ps}$ is traced and extracted with respect to the rotation angle $\theta$ from the $x$-axis. In the figure showing the extracted information, the radius normalized by the average radius of $19.87 \mathrm{~nm}$ is plotted. It was confirmed that there are preferential growth directions at rotation angles of $0^{\circ}, 90^{\circ}$, $180^{\circ}$ and $270^{\circ}$, which correspond to the $\langle 100\rangle$ direction. This is in agreement with the information 
in Table I, in which the kinetic coefficient of the $\langle 100\rangle$ direction is larger than that of the $\langle 110\rangle$ direction. The spontaneous evolution of anisotropy in a solid nucleus during solidification was achieved for the first time with the aid of the high processing ability of the GPU architecture.

\section{Homogeneous Nucleation and Subsequent Grain Growth}

One of the remaining issues in the simulation of solidification is how to treat the nucleation. ${ }^{54}$ In existing phase-field simulations, the nuclei in the melt are given in advance with a random distribution or are formed forcibly on the basis of classical nucleation theory. On the other hand, it is possible in principle to achieve nucleation in molecular dynamics simulations when suitable conditions are given. For example, nucleation in a nanoscale liquid droplet has been achieved with relative ease under continuous cooling, ${ }^{61,62}$ which has been widely examined to study the size dependence of the melting point of metal nanoparticles. ${ }^{61-66}$ However, generally, it is not yet straightforward to achieve multiple nucleation, which is essential for the formation of polycrystalline microstructures, since a broad range of temporal and spatial scales is required. Therefore, multiple nucleation in a large-scale system is usually achieved with the aid of inducing factors such as a high pressure ${ }^{53}$ and surface fluctuation. ${ }^{67}$

We successfully achieved spontaneous nucleation from an undercooled iron melt without any inducing factor in a million-atom molecular dynamics simulation on a GPU supercomputer using the code described in the previous subsection. The simulation methodology basically followed the simulation in the previous subsection. Firstly, a bcc crystal of iron with a size of $53.4 \times 53.4 \times 4.3 \mathrm{~nm}^{3}(1,037,880$ atoms) was heated at $3500 \mathrm{~K}$ under a NVT (number of atoms, volume and temperature) constant condition to obtain an iron melt as the initial configuration. The prepared initial configuration was isothermally undercooled at $\Delta T=1000 \mathrm{~K}$ for 10,000 ps under zero pressure by a NPT (number of atoms, pressure and temperature) constant condition. The Finnis-Sinclair potential ${ }^{57}$ was employed for the interaction between iron atoms as in the above simulation. The periodic boundary condition was employed for all boundaries. Figure 2 shows snapshots of the atomic configuration during a consecutive simulation of nucleation, solidification and grain growth. Many nuclei are simultaneously nucleated before $150 \mathrm{ps}$ and grow to form spherical grains in the melt. Other nuclei are continuously nucleated from the remaining melt. Later-nucleated grains fill the spaces between earlier-nucleated grains, and all the iron melt has solidified by 300 ps. After the solidification, the small grains gradually shrink and disappear whereas the large ones become larger, which is regarded as grain coarsening. Since the existence of grain boundaries yields excess grain boundary energy (approximately $0.5 \mathrm{~J} / \mathrm{m}^{2}$ to $2.0 \mathrm{~J} / \mathrm{m}^{260}$, such grain growth occurs in order to decrease the area of grain boundaries. It was also confirmed from the molecular dynamics simulation that the rate of grain coarsening is one order of magnitude slower than that of the solidification.

The incubation time until the first nucleation and the number of nuclei drastically change when the undercooling temperature is varied. It was confirmed that the incubation time as a function of temperature has a peak (i.e., nose shape) at the critical temperature, which is a characteristic shape of the time-temperature-transformation (TTT) curve. ${ }^{54}$ Therefore, it is considered that the nucleation observed in Fig. 2 is entirely thermally activated without any other inducing factor. The thermally activated nucleation in the million-atom molecular dynamics simulation has been investigated in detail elsewhere. ${ }^{54}$

\section{ADVANCES IN QUANTITATIVE COMPUTATION OF SOLIDIFICATION MICROSTRUCTURES}

\section{Quantitative Phase-Field Model}

As seen above, recent atomic-scale simulations can capture the scale of microstructure evolution. On the other hand, the description and prediction of microstructural evolution during solidification have generally been theoretically and numerically tackled within the framework of a free-boundary problem, the underlying physics of which are solutal and thermal diffusion in the bulk, mass and energy conservation laws at the interface and the GibbsThomson effects. One of the central issues in modeling microstructural processes is therefore the precise description of the interface dynamics consistent with the free-boundary problem. The phasefield model has emerged as a powerful tool for describing the microstructural evolution processes. ${ }^{18-23}$ This is a diffuse interface approach, in which the interface is not sharp but diffuse, exhibiting non-zero thickness. The main advantage of this model is that it is not necessary to explicitly track the position of a sharp interface in complex microstructural patterns. The phase-field model has been applied to a variety of solidification processes, ${ }^{18-22}$ and its capability of affording a qualitative understanding of phenomena has generally been acknowledged. Despite this success, however, a long-standing issue regarding the quantitative aspect of the phase-field model remained unresolved until recently.

Phase-field models were developed in early works to reproduce the free-boundary problem of interest in the so-called sharp-interface limit, where the thickness of the diffuse interface $W$ approaches zero. However, in practice, a prerequisite for this diffuse interface approach is to assign a finite value to $W$. A realistic value of $W$ for the solid-liquid interface is typically a few $\mathrm{nm}$; thus, a spatial resolution of $\AA$ 


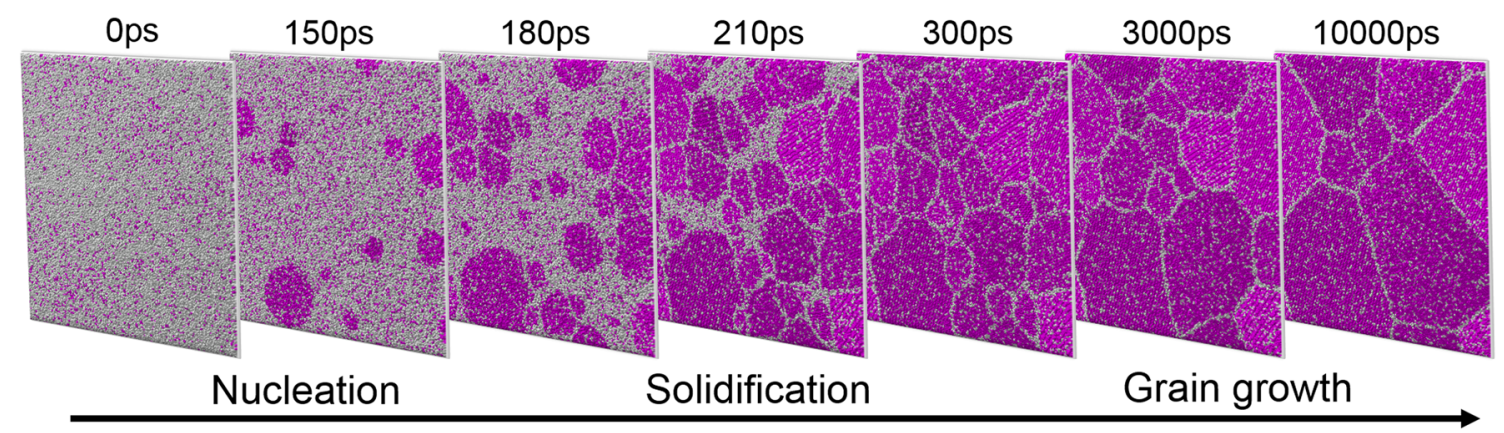

Fig. 2. Consecutive molecular dynamics simulation of nucleation, solidification and grain growth. An iron melt consisting of $1,037,880$ atoms in a cell of $53.4 \times 53.4 \times 4.3 \mathrm{~nm}^{3}$ was isothermally undercooled at $\Delta T=1000 \mathrm{~K}$ over $10,000 \mathrm{ps}$. Purple and white atoms represent iron atoms with and without the bcc configuration, respectively.

order is required to describe a diffuse interface having a realistic thickness. This high spatial resolution limits the system size to extremely small, making it impossible to deal with problems at the microstructural scale. Therefore, $W$ has to be increased by orders of magnitude from the realistic thickness. However, this increment, in turn, causes the unrealistic magnification of some physical effects associated with the diffuse interface, which precludes the quantitative computation of solidification microstructures.

This serious problem was resolved by Karma and Rappel. ${ }^{24,25}$ They put forward a model based on a new procedure called the thin-interface limit, in which $W$ is taken to be smaller than any physical length appearing at the microstructural scale but much larger than the realistic thickness. This model is called the quantitative phase-field model since it enables quantitatively meaningful simulations. ${ }^{25}$ This model was later extended to deal with alloy solidification in a dilute binary alloy with zero diffusivity in the solid (one-sided model). ${ }^{26,27}$ Moreover, the quantitative phase-field model has been developed to describe two-phase solidification in binary alloys with zero diffusivity in the solids (onesided model) ${ }^{28}$ alloy solidification with coupled heat and solute diffusion in dilute binary alloys having zero solutal diffusivity in the solid and equal thermal diffusivities in the solid and liquid (one-sided solute transport and symmetric heat transport), ${ }^{29}$ and isothermal solidification in multicomponent alloys with zero diffusivities in the solid (one-sided model).$^{30}$ In addition, one of the present authors has recently developed quantitative phase-field models for the two-sided case. To be more specific, the models were developed for isothermal single-phase solidification, ${ }^{31}$ two-phase solidification in dilute binary alloys with an arbitrary value of the solid diffusivity, ${ }^{32}$ and single-phase solidification in multicomponent alloys with coupled solutal and thermal diffusion. ${ }^{33}$ These two-sided models enable us to describe the equilibrium solidification, the microsegregation, the motion of the solid-solid interface, and the solidification processes in practical alloy systems such as carbon steels, where the dif- fusion in the solid is not negligible. Quantitative phase-field models are being increasingly utilized for quantitative simulations of solidification phenomena. ${ }^{34,36,68-81}$

As mentioned above, significant progress has been made in quantitative phase-field modeling for alloy solidification. The accuracy of quantitative phase-field simulations is evaluated by observing the convergence behavior of the simulation results with decreasing $W$. It has been demonstrated that the convergence of the results in quantitative phase-field simulations is much faster than that of the results in the conventional phase-field model, ${ }^{31-33}$ which indicates that accurate results can be obtained using a large value for $W$ in quantitative phase-field models. This is quite advantageous in terms of the computational cost because the computational time for a three-dimensional simulation using a finite difference method is proportional to $W^{-5}{ }^{69}$ Hence, the quantitative phase-field model enables highly accurate and large-scale computations of solidification microstructures. However, it should be pointed out that the value of $W$ required to obtain well-converged results is strongly dependent on the solidification condition of interest. No criterion has yet been established regarding a suitable choice of $W$, and a convergence test is generally required for each solidification condition to ensure accurate and efficient computations. Therefore, it is desirable to obtain information to reduce the effort involved in the convergence test. This point is addressed below.

\section{Convergence of Outcome in Quantitative Phase-Field Simulation}

We have carried out quantitative phase-field simulations of the directional solidification of Al-2mass $\% \mathrm{Cu}$ alloy in a two-dimensional system to gain some insight into the convergence behavior. The competitive growth of solids was analyzed by considering a single solid growing in the $y$-direction with the periodic boundary condition applied in the $x$-direction. The details of time evolution equations can be found in Ref. 36. The input parameters used 
in this study are listed in Table II. A temperature gradient and an initial undercooling were set to $G=1000 \mathrm{~K} / \mathrm{m}$ and $u_{0}=\left(c_{1}-c_{0}\right) /\left[(1-k) c_{0}\right]=-1$, respectively, where $c_{1}$ is the concentration in the liquid phase, $c_{0}$ is the average concentration, and $k$ is the partition coefficient. We started with initial seeds periodically spaced by the targeted spacing. This spacing corresponds to the primary arm spacing $\lambda$ and it was set to about $150 \mu \mathrm{m}$. By performing a moving frame simulation, we obtained steady-state values of tip undercooling, given by $\Omega=1-y_{\text {tip }} /$ $l_{\mathrm{T}}$, where $y_{\text {tip }}$ is the position of the dendrite tip and $l_{\mathrm{T}}$ is the thermal diffusion length, and the curvature radius of the dendrite tip $\rho$.

The calculated results for $V=500$ and $50 \mu \mathrm{m} / \mathrm{s}$ are shown in Fig. $3 \mathrm{a}$ and b, respectively. The horizontal axis is the interface thickness normalized by the chemical capillary length $d_{0}$. In each case, $W$ and $\rho$ fully converge with unique values when $W$ is small, which indicates excellent convergence behavior. However, the value of $W$ required for the convergence strongly depends on the pulling velocity, $V$. The convergence for $V=500$ and $50 \mu \mathrm{m} / \mathrm{s}$ starts to break down when $W / d_{0}<60$ and 200 , respectively. This large difference in the convergence behavior indicates that the effort required to find a suitable value of $W$ strongly depends on the solidification condition of interest. One may suppose that the breakdown of convergence should be related to the onset of the unphysical magnification of the interface effects that always exists in conventional phase-field models constructed in the sharpinterface limit as described in the previous section.

According to Fig. $3 a$ and $b$, the converged value of $\rho$ is strongly dependent on the pulling velocity. Hereafter, the values of $\Omega$ and $\rho$ calculated for the smallest value of $W$ are regarded as the converged values and are, respectively, denoted by $\Omega_{\mathrm{c}}$ and $\rho_{\mathrm{c}}$. In Fig. 3, the values of $\rho_{\mathrm{c}} / d_{0}$ for $V=500$ and $50 \mu \mathrm{m} / \mathrm{s}$ are about 300 and 1000 , respectively. Hence, from the comparison between Fig. $3 a$ and $b$, one may speculate that the convergence of the simulation for a relatively coarse structure starts to break down at a relatively large $W$. We investigated the validity of this speculation in a quantitative manner. All the data shown in Fig. $3 \mathrm{a}$ and $\mathrm{b}$ are plotted in Fig. 3c, where $\rho$ and $\Omega$ are normalized by $\rho_{\mathrm{c}}$ and $\Omega_{\mathrm{c}}$, re-

Table II. Input parameters employed in quantitative phase-field simulations for directional solidification of an $\mathrm{Al}-2 \mathrm{mass} \% \mathrm{Cu}$ alloy ${ }^{36}$

Melting temperature of pure $\mathrm{Al}\left(T_{\mathrm{m}}\right)$

$933.25 \mathrm{~K}$

Liquidus slope $(m)$

Partition coefficient $(k)$

Gibbs-Thomson coefficient $(\Gamma)$

Strength of anisotropy of

solid-liquid interface $(\varepsilon)$

Liquid diffusivity $\left(D_{1}\right)$

Solid diffusivity $\left(D_{\mathrm{s}}\right)$

20 (K/at. frac.) 0.14

$2.4 \times 10^{-7}(\mathrm{~K} \mathrm{~m})$

0.02

$3 \times 10^{-9}\left(\mathrm{~m}^{2} / \mathrm{s}\right)$

$2 \times 10^{-12}\left(\mathrm{~m}^{2} / \mathrm{s}\right)$ spectively, on the $y$-axis and $W$ is normalized by $\rho_{\mathrm{c}}$ on the $x$-axis. It can be seen that the convergence starts to break down when $W / \rho_{\mathrm{c}} \sim 0.2$ in both cases. In other words, regardless of the solidification condition, the results fully converge as long as $W /$ $\rho_{\mathrm{c}} \leq 0.2$. This is also supported by the results for the directional solidification of an impure succinonitrile alloy in Ref. 27.

Note that the steady-state profile of the phasefield in our model is given by $\phi=\tanh \left[r /\left(2^{1 / 2} W\right)\right]$. Here, $\phi$ is the phase-field, which takes a value of +1 $(-1)$ in the solid (liquid) and continuously changes from -1 to +1 inside the interface, and $r$ is the spatial coordinate in the direction normal to the interface. This solution is obtained for the boundary condition $\phi= \pm 1$ at $r \rightarrow \pm \infty$. In this model, the interface thickness cannot be well defined. In the above discussion, $W$ was used as a measure of the interface thickness and is actually the length of the region for $-0.34<\phi<0.34$. When the region for $-0.95<\phi$ $<0.95$ is considered, the thickness of this region $W^{\prime}$ is about $5 \mathrm{~W}$. Hence, the condition $W / \rho_{\mathrm{c}} \leq 0.2$ corresponds to $W^{\prime} \leq \rho_{\mathrm{c}}$. Within the framework of the diffuse interface approach, an accurate description of the size and morphology of microstructures is not possible when the interface thickness is set to larger than the minimum curvature radius of the interface appearing in the microstructure. The condition $W^{\prime} \leq \rho_{\text {c }}$ should originate from this fact. Namely, the breakdown of the convergence shown in Fig. 3c is not triggered by the onset of the unphysical magnification of interface effects and is actually a natural consequence of the limitation unique to the diffuse interface approach.

To provide evidence for this, data for free dendritic growth reported in the literature ${ }^{31,32}$ are plotted in Fig. 4 in the same manner as in Fig. 3c. Six sets of data are distinguished by symbols with different shapes. For each dataset, the open and filled symbols represent $V / V_{\mathrm{c}}$ and $\rho / \rho_{\mathrm{c}}$, respectively. Here, $V$ is the steady-state value of moving velocity of dendrite tip and $V_{c}$ is the converged one, viz., the value calculated for the smallest value of $W$. Datasets A and B are the results for isothermal solidification in binary alloys (Figs. 4 and 5 in Ref. 31). Datasets C and D are those for nonisothermal solidification in a binary alloy without and with diffusion in the solid (Figs. 2 and 3 in Ref. 32) and datasets $\mathrm{E}$ and $\mathrm{F}$ are the results for isothermal and non-isothermal solidification in a ternary alloy (Figs. 4 and 5 in Ref. 32), respectively. Importantly, all the data converge as long as $W / \rho_{\mathrm{c}} \leq 0.2$. Hence, the condition $W / \rho_{\mathrm{c}} \leq 0.2$ holds true in both directional and free dendritic growth. This fact will reduce the effort involved in convergence tests. Once the minimum curvature radius, $\rho_{\mathrm{c}}$, of the growing phase(s) appearing during a microstructural evolution process is obtained from preliminary simulations, accurate and efficient computation can be conducted by assigning a value of about $0.2 \rho_{\mathrm{c}}$ to $W$. 


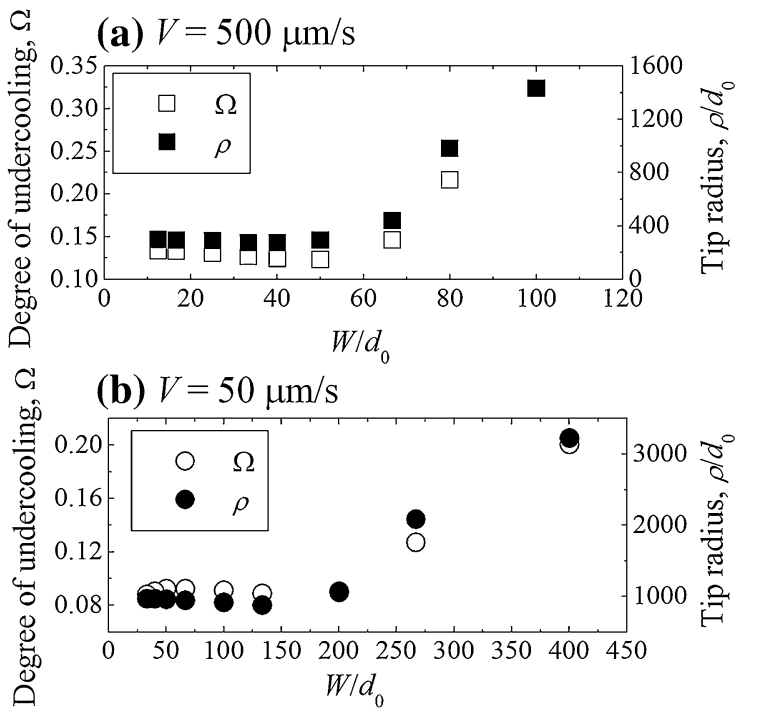

(c)

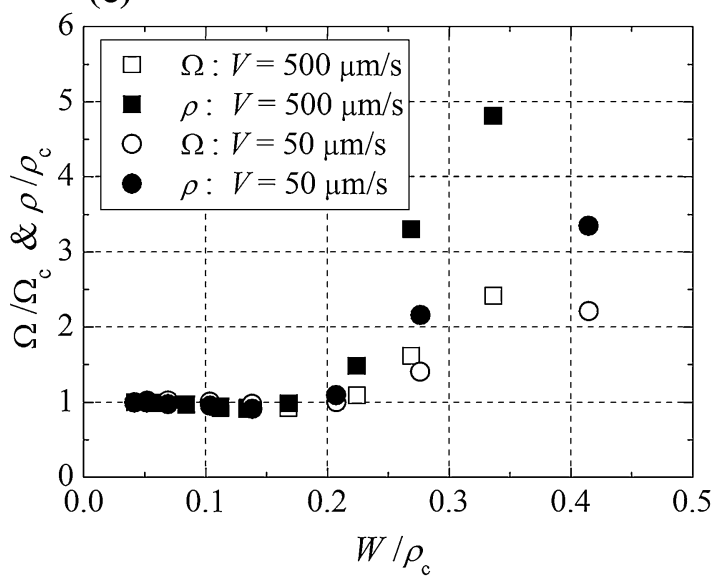

Fig. 3. Convergence behavior of degree of undercooling and curvature radius of the dendrite tip during directional dendritic growth in Al$2 \mathrm{mass} \% \mathrm{Cu}$ alloy calculated for (a) $V=500$ and (b) $50 \mu \mathrm{m} / \mathrm{s}$. (c) Convergence behavior plotted on normalized axes.

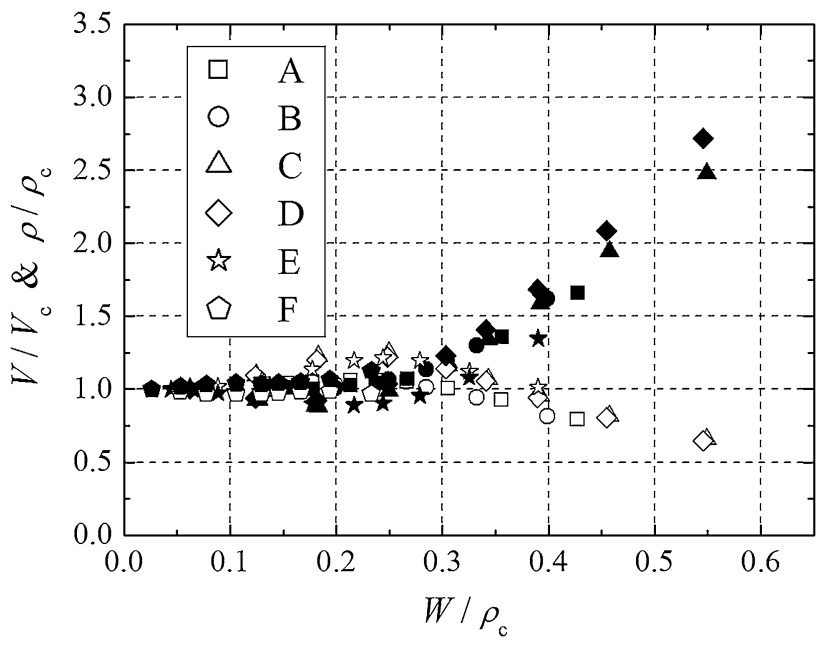

Fig. 4. Convergence behavior of velocity (open symbols) and curvature radius (filled symbols) of the dendrite tip during free dendritic growth in binary $(A, B, C, D)$ and ternary (E, F) alloys. Details of datasets $A-F$ can be found in the text.

\section{LARGE-SCALE PHASE-FIELD SIMULATION OF COMPETITIVE GROWTH OF DENDRITE ASSEMBLAGES}

\section{High-Performance Computation for the Phase-Field Method}

The development of the quantitative phase-field model enables the use of a large interface thickness $W$ or a large computational lattice. However, as shown in the previous section, the value of $W$ is restricted by the curvature of the dendrite tip $\rho$. Therefore, dendrite growth simulations using the phase-field method have been limited to two-dimensional problems or three-dimensional simula- tions of a small number of dendrites. Actually, many solidification structures are formed through the interactions during the competitive growth of dendrite assemblages. ${ }^{1,2}$ Although the cellular automaton method has been widely used for polycrystal solidification simulations, ${ }^{15,16,82}$ and has been employed for large-scale solidification simulations, ${ }^{83,84}$ multiple-dendrite-growth simulation by the phase-field method is crucial for accurate prediction of the solidification microstructure. An adaptive mesh refinement technique, in which fine meshes are used only around the interface,${ }^{85-89}$ can reduce the computational cost. However, its applicability to polycrystal solidification with a large interface area fraction is not flexible. Moreover, the development of the code for adaptive mesh refinement requires tremendous effort.

Under such circumstances, GPU computation has attracted the attention of many phase-field researchers because GPUs have been successfully used to increase the speed of phase-field computation. ${ }^{33,90}$ Moreover, parallel computation using multiple GPUs has the potential to capture realistic dendrite assemblages. ${ }^{22,35,91,92}$ Shimokawabe et al. achieved the first-ever petascale phase-field simulation of dendrite growth using 4000 GPUs on the TSUBAME2.0 supercomputer at the Tokyo Institute of Technology. ${ }^{92}$ Subsequently, the present authors and coworkers successfully performed a very-large-scale simulation of multiple dendritic competitive growth using $4000^{3}$ meshes. ${ }^{35}$

From the viewpoint of applications, understanding of the competitive growth of dendrite assemblages is essential to improve and control solidification microstructures. It is widely accepted that dendrites whose $\langle 100\rangle$ preferential growth direction is almost parallel to the heat flow direction can continue to grow by stopping the growth of 

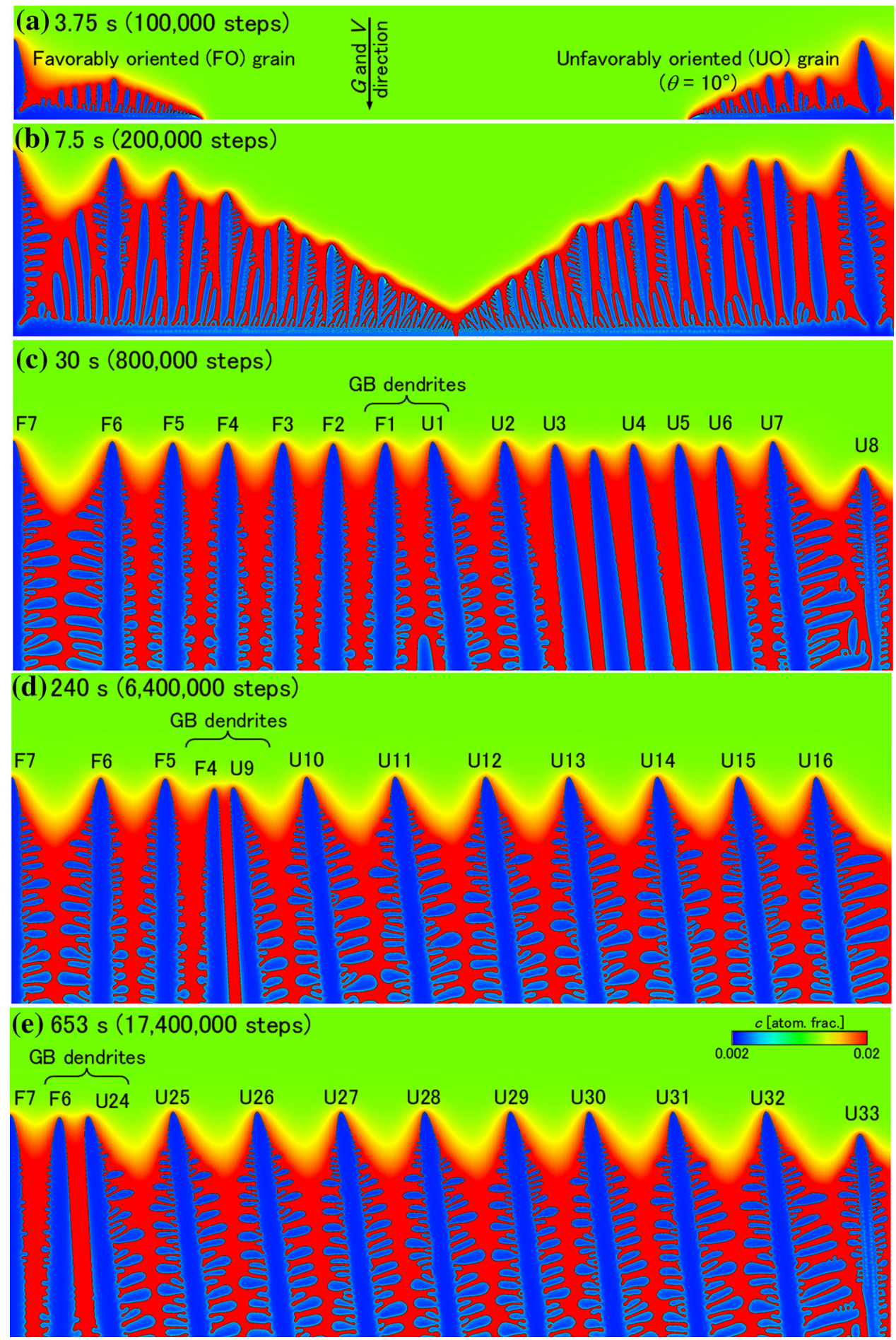

Fig. 5. Two-dimensional simulation of competitive growth of Al-3mass $\% \mathrm{Cu}$ binary alloy bicrystal with $V=100 \mu \mathrm{m} / \mathrm{s}$ and $\mathrm{G}=10 \mathrm{~K} / \mathrm{mm}$. The domain size was set to $3.072 \times 1.152 \mathrm{~mm}^{2}(4096 \times 1536$ meshes $)$ and the time step was $\Delta t=3.75 \times 10^{-5} \mathrm{~s}$. Zero Neumann boundary conditions for $\phi$ and $u$ were set for all boundaries.

dendrites having a $\langle 100\rangle$ crystallographic orientation that deviates from the heat flow direction. ${ }^{16,93,94}$ On the other hand, unusual dendrite selections, in which inclined dendrites overgrow dendrites growing in the heat flow direction, have recently been observed in the unidirectional solidification of a bicrystal sample. ${ }^{95,96}$ To clarify the mechanism of this unusual overgrowth, two-dimensional phase-field simulations have been performed..$^{34,36,90}$ In the following subsections, we 

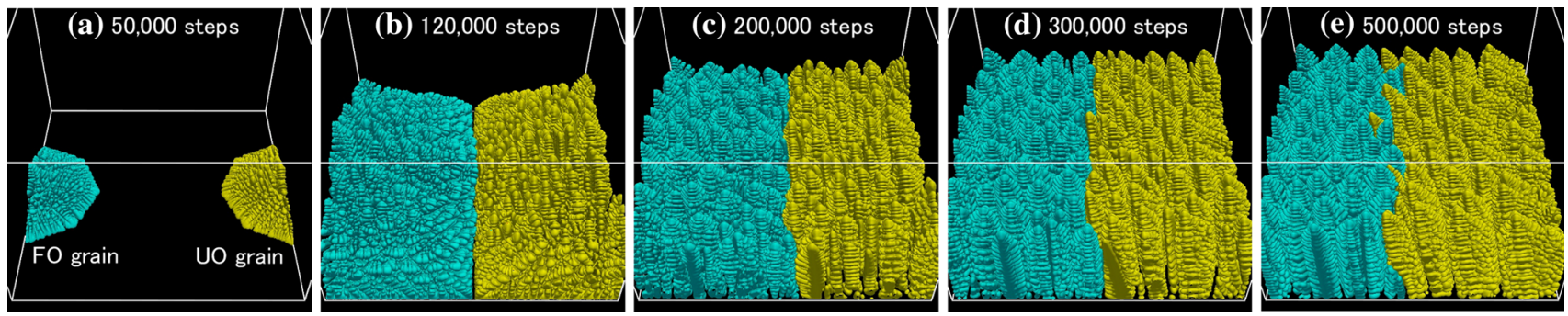

Fig. 6. Three-dimensional simulation of competitive growth of Al-3mass $\% \mathrm{Cu}$ binary alloy bicrystal with $V=100 \mu \mathrm{m} / \mathrm{s}$ and $\mathrm{G}=10 \mathrm{~K} / \mathrm{mm}$. The domain size was set to $1.536 \times 1.536 \times 1.024 \mathrm{~mm}^{3}(1536 \times 1536 \times 1024$ meshes $)$ and the time step was $\Delta t=4.74 \times 10^{-5} \mathrm{~s}$. Zero Neumann boundary conditions for $\phi$ and $u$ were set for all boundaries. The sky blue and yellow grains are the favorably and unfavorably oriented grains, respectively.

report the competitive growth of dendrite assemblages investigated by two- and three-dimensional simulations using the quantitative phase-field model, which were performed on multiple GPUs.

\section{Two-Dimensional Simulation of Competitive Growth}

Figure 5 shows snapshots from a two-dimensional simulation of competitive dendrite growth during the directional solidification of a binary alloy bicrystal. The quantitative phase-field model for the solidification of a dilute binary alloy ${ }^{31}$ was used with the moving frame algorithm for directional solidification under a constant temperature gradient, $G$. The computational conditions were same as in Ref. 36 except for a computational domain of $3.072 \times 1.152 \mathrm{~mm}^{2}(4096 \times 1536$ meshes $)$ and 20 million computational steps (750 s) performed for Al-3mass\%Cu with $V=100 \mu \mathrm{m} / \mathrm{s}$ and $G=10 \mathrm{~K} / \mathrm{mm}$. The computation was performed within 1 day using eight GPUs. Two seeds were placed at the two bottom corners of the computational domain, where the left seed was the favorably oriented (FO) grain and the right seed was the unfavorably oriented (UO) grain with its $\langle 100\rangle$ crystallographic orientation at angle of $10^{\circ}$ from the heat flow direction. As shown in Fig. 5a, the solids cover the bottom surface and the dendrites grow in the heat flow direction. A grain boundary (GB) is formed at the collision point between the two grains, as shown in Fig. 5b, and steady-state competitive growth between the GB dendrites starts from $8 \times 10^{5}$ steps (Fig. 5c). Here, $\mathrm{FO}$ and UO dendrites are labeled using "F" and "U", respectively. At the GB, some UO dendrites are blocked by the FO dendrite, and then the UO dendrite overgrows the FO dendrite after the blocking. Finally, all the FO dendrites are overgrown after about 18 million steps. To overgrow the FO dendrites labeled F1-F7, 3, 3, 3, 3, 8, 9 and 1 UO dendrites are required, respectively. This means that, for example, the F1 dendrite blocks the growth of the $\mathrm{U} 1$ and $\mathrm{U} 2$ dendrites and is overgrown by the U3 dendrite, and the F2 dendrite blocks the U3 and U4 dendrites and is overgrown by the U5 dendrite. This difference is mainly caused by the difference in the dendrite arm spacing between the GB FO den- drite and the FO dendrite at its immediate left. As shown in Fig. 5c, the arm spacing of F5-F6 $(197 \mu \mathrm{m})$ and $\mathrm{F} 6-\mathrm{F} 7(324 \mu \mathrm{m})$ is the largest compared to F1-F2 $(175 \mu \mathrm{m}), \mathrm{F} 2-\mathrm{F} 3(161 \mu \mathrm{m}), \mathrm{F} 3-\mathrm{F} 4$ $(182 \mu \mathrm{m})$, and F4-F5 $(179 \mu \mathrm{m})$. In addition, the UO dendrite arm spacing also affects the overgrowth of FO dendrites. The F4 dendrite is overgrown by the U9 dendrite. The average UO dendrite arm spacing shown in Fig. 5c, or U1-U7, is $199 \mu \mathrm{m}$. On the other hand, the average UO dendrite arm spacing shown in Fig. 5d, or U10-U16, is $296 \mu \mathrm{m}$. Thus, the large difference in the number of UO dendrites needed to overgrow the $\mathrm{FO}$ dendrite is caused by both spacing of FO dendrites and UO dendrites. As shown in Fig. 5d, when the U9 dendrite approaches the F4 dendrite, both GB dendrites fall down and the F4 dendrite moves to the left. When the spacing between F4 and F5 reaches the minimum value in which the two dendrites can coexist, ${ }^{36}$ the F4 dendrite is overgrown by the U9 dendrite. Accordingly, the horizontal migration of the FO dendrite when the UO dendrite approaches to the FO dendrite is a key process in the unusual selection. The unusual overgrowth observed in the present simulation occurs less readily with increasing inclination angle of the UO dendrites ${ }^{90}$ and pulling velocity ${ }^{34}$ due to the reduced the solute interaction around the tips of the GB dendrites.

\section{Three-Dimensional Simulation of Competitive Growth}

As introduced in the previous section, the basic mechanism of the unusual dendrite selection can be investigated by two-dimensional simulation. However, actual dendrite growth occurs in three-dimensional space and the competition between dendrites at GBs is more complicated. ${ }^{97,98}$ Figure 6 shows the snapshots from a three-dimensional simulation of competitive dendrite growth during the directional solidification of a binary alloy bicrystal. The computational domain was set to $1.536 \times 1.536 \times 1.024 \mathrm{~mm}^{3} \quad(1536 \times 1536 \times 1024$ meshes) and a computation of 0.5 million steps $(23.7 \mathrm{~s})$ was performed. Except for the lattice size of $\Delta x=1 \mu \mathrm{m}$ and the inclination angle of UO grain of $20^{\circ}$, the computational conditions were the same as 


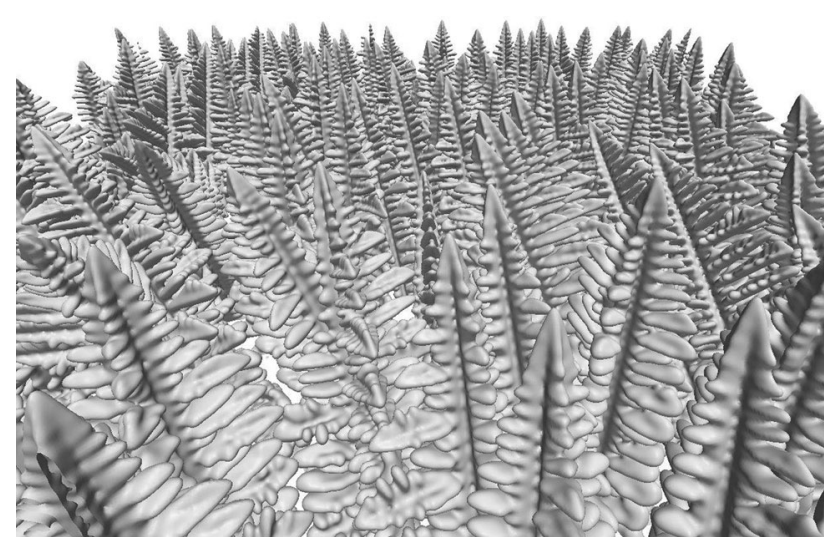

Fig. 7. Snapshot from a very-large-scale three-dimensional phasefield simulation of the directional solidification of a binary alloy polycrystal. ${ }^{35}$ The calculation were carried out in a system with dimensions of $3.072 \times 3.072 \times 3.072 \mathrm{~mm}^{3} \quad(4096 \times 4096 \times 4096$ meshes) for a total time period of more than $100 \mathrm{~s}$ (4 million computational steps) using 768 GPUs with 768 CPUs on TSUBAME2.0.

in the previous two-dimensional simulation. It took about half a day for this simulation to be performed using 512 GPUs of the TSUBAME2.5 supercomputer at Tokyo Institute of Technology, which is a practical computational time. At the beginning of the computation, as shown in Fig. 6a, the two grains spread along the bottom surface to form a fanlike shape, and many secondary arms grow in the heat flow direction. Figure $6 \mathrm{~b}$ show that the two grains collide and a straight GB is formed because of the high density of arms. Dendrite selection subsequently occurs and the number of dendrites decreases as shown in Fig. $6 \mathrm{c}$ and d. Because the inclined dendrites grow in the $\langle 100\rangle$ direction with increasing arm spacing, ${ }^{99-102}$ the UO dendrites move toward the FO dendrites. As a result, the competition between dendrites becomes intense at the GB, and the shape of the GB becomes zigzag as shown in Fig. 6e. This zigzag GB is very similar to that observed experimentally. ${ }^{97,98}$

Here, we showed the very beginning stage of competitive growth. By continuing this simulation longer, we will be able to observe the detail competition between FO and UO dendrites in three-dimensional space in detail. In three-dimensional space, because the solute diffusion is possible in the three directions, we need a longer computational time than for the two-dimensional problem to see the unusual overgrowth phenomenon. Therefore, this is challenging topic even using a supercomputer. Nevertheless, the results will be available in the near future.

\section{CONCLUSION}

Utilizing the high parallel efficiency of GPUs, cutting-edge simulations were performed to capture the nature of solidification from various viewpoints. From an atomic viewpoint, a million-atom molecular dynamics simulation revealed the spontaneous evolution of anisotropy in a solid nucleus embedded in an undercooled iron melt, in which fourfold symmetry was achieved naturally without the use of any empirical parameters. Homogeneous nucleation from an undercooled melt was achieved by another million-atom molecular dynamics simulation, in which multiple nuclei solidified to form a multigrain microstructure and grain coarsening occurred during $10 \mathrm{~ns}$, according to the results of the calculation. Moreover, the convergence behavior in quantitative phase-field simulations has been discussed in detail. Such convergence enables the use of a large interface thickness in quantitative phase-field simulations. Using the quantitative phase-field model for the solidification of a dilute binary alloy, the competitive growth of dendrite assemblages during the directional solidification of a binary alloy bicrystal in a millimeter scale was examined by performing two- and three-dimensional large-scale simulations by multiGPU computation. From the two-dimensional simulation, the mechanism of the unusual overgrowth phenomenon, in which dendrites inclined to the heat flow direction overgrow those growing in the heat flow direction during unidirectional solidification, was clarified. On the other hand, a zigzag grain boundary was formed during the competition between favorably and unfavorably oriented dendrites in the three-dimensional phasefield simulation.

In summary, many topics remain to be investigated in solidification science and other fields of metallurgy. We believe that large-scale simulations are powerful tools for their investigation and should bring about significant changes in computational metallurgy. Although results from molecular dynamics and phase-field simulations in this paper are not directly linked but so far independent, further large-scale molecular simulation will enable a direct comparison with the phase-field and other mesoscale simulations. Moreover, the statistical sampling of nucleation in the large-scale molecular dynamics simulation can export the proper information of nucleation event to the phase-field simulation in the near future. Finally, we celebrate the beginning of a new phase of computational metallurgy with the impressive snapshot in Fig. 7, which was obtained from a very-large-scale threedimensional phase-field simulation of the directional solidification of a binary alloy polycrystal. ${ }^{35}$ The calculation was carried out in a system with dimensions of $3.072 \times 3.072 \times 3.072 \mathrm{~mm}^{3}$ $(4096 \times 4096 \times 4096$ meshes $)$ for a total time period of more than $100 \mathrm{~s}$ (4 million computational steps) using 768 GPUs with 768 CPUs on the TSUBAME2.0, which is the largest simulation of dendrite growth ever to be reported to the best of our knowledge. 


\section{ACKNOWLEDGEMENTS}

This work was supported by Grant-in-Aid for Scientific Research (B) (Nos. 25289266 and 25289006) from Japan Society for the Promotion of Science (JSPS), Japan; 22th (T.T and M.O) and 23th (Y.S) ISIJ Research Promotion Grants from the Iron and Steel Institute of Japan (ISIJ), the Strategic Programs for Innovative Research (SPIRE), MEXT; and the Computational Materials Science Initiative (CMSI), Japan. Part of this work was supported by the Joint Usage/Research Center for Interdisciplinary Large-scale Information Infrastructures (JHPCN) and the High Performance Computing Infrastructure (HPCI) in Japan for the computational environment. Y.S. would like to thank Ms. Kanae Oguchi for support in the development of the source code for the GPU environment for the molecular dynamics simulations. T.T. also thanks Mr. Shinji Sakane, Prof. Takashi Shimokawabe, and Prof. Takayuki Aoki for the support in the largescale simulations using the TSUBAME2.5 supercomputer.

\section{OPEN ACCESS}

This article is distributed under the terms of the Creative Commons Attribution 4.0 International License (http://creativecommons.org/licenses/by/4.0/ ), which permits unrestricted use, distribution, and reproduction in any medium, provided you give appropriate credit to the original author(s) and the source, provide a link to the Creative Commons license, and indicate if changes were made.

\section{REFERENCES}

1. W. Kurz and D.J. Fisher, Fundamentals of Solidification (Aedermannsdorf: Trans Tech Publications, 1998), pp. 116.

2. J.A. Dantzig and M. Rappaz, Solidification (Lausanne: EPFL Press, 2009), pp. 1-22.

3. K.A. Jackson and J.D. Hunt, Acta Metall. 13, 1212 (1965).

4. H. Esaka and W. Kurz, J. Cryst. Growth 72, 578 (1985).

5. R.H. Mathiesen, L. Arnberg, F. Mo, T. Weitkamp, and A. Snigirev, Phys. Rev. Lett. 83, 5062 (1999).

6. A. Bogno, H. Nguyen-Thi, A. Buffet, G. Reinhart, B. Billia, N. Mangelinck-Noël, N. Bergeon, J. Baruchel, and T. Schenk, Acta Mater. 59, 4356 (2011).

7. H. Yasuda, T. Nagira, M. Yoshiya, A. Sugiyama, N. Nakatsuka, M. Kiire, M. Uesugi, K. Uesugi, K. Umetani, and K. Kajiwara, IOP Conf. Ser.: Mater. Sci. Eng. 33, 012036 (2012).

8. R.B. Potts, Math. Proc. 48, 106 (1952).

9. D.J. Srolovitz, M.P. Anderson, G.S. Grest, and P.S. Sahni, Scr. Metall. 17, 241 (1983).

10. M.P. Anderson, D.J. Srolovitz, G.S. Grest, and P.S. Sahni, Acta Metall. 32, 783 (1984).

11. A.W. Godfrey and J.W. Martin, Philos. Mag. A 72, 737 (1995).

12. T. Koseki, H. Inoue, Y. Fukuda, and A. Nogami, Sci. Technol. Adv. Mater. 4, 183 (2003).

13. D. Juric and G. Tryggvason, J. Comput. Phys. 123, 127 (1996).

14. A. Jacot and M. Rappaz, Acta Mater. 50, 1909 (2002).

15. M. Rappaz and C.A. Gandin, Acta Metall. Mater. 41, 345 (1993).

16. C.A. Gandin and M. Rappaz, Acta Metall. Mater. 42, 2233 (1994).
17. R. Kobayashi, Phys. D 63, 410 (1993).

18. W.J. Boetinger, J.A. Warren, C. Beckermann, and A. Karma, Annu. Rev. Mater. Res. 32, 163 (2002).

19. L. Granasy, T. Pusztai, and J.A. Warren, J. Phys.: Condens. Matter. 16, R1205 (2004).

20. H. Emmerich, Adv. Phys. 57, 1 (2008).

21. I. Steinbach, Model. Simul. Mater. Sci. Eng. 17, 073001 (2009).

22. T. Takaki, ISIJ Int. 54, 437 (2014).

23. S.G. Kim, W.T. Kim, and T. Suzuki, Phys. Rev. E 60, 7186 (1999).

24. A. Karma and W.-J. Rappel, Phys. Rev. E 53, R3017 (1996).

25. A. Karma and W.-J. Rappel, Phys. Rev. E 57, 4323 (1998).

26. A. Karma, Phys. Rev. Lett. 87, 115701 (2001).

27. B. Echebarria, R. Folch, A. Karma, and M. Plapp, Phys. Rev. E 70, 061604 (2004)

28. R. Folch and M. Plapp, Phys. Rev. E 72, 011602 (2005).

29. J.C. Ramirez, C. Beckermann, A. Karma, and H.-J. Diepers, Phys. Rev. E 69, 051607 (2004).

30. S.G. Kim, Acta Mater. 55, 4391 (2007).

31. M. Ohno and K. Matsuura, Phys. Rev. E 79, 031603 (2009).

32. M. Ohno and K. Matsuura, Acta Mater. 58, 5749 (2010).

33. M. Ohno, Phys. Rev. E 86, 051603 (2012).

34. J. Li, Z. Wang, Y. Wang, and J. Wang, Acta Mater. 60, 1478 (2012).

35. T. Takaki, T. Shimokawabe, M. Ohno, A. Yamanaka, and T. Aoki, J. Cryst. Growth 382, 21 (2013).

36. T. Takaki, M. Ohno, T. Shimokawabe, and T. Aoki, Acta Mater. 81, 272 (2014).

37. R. Tönhardt and G. Amberg, J. Cryst. Growth 213, 161 (2013).

38. T. Takaki, R. Rojas, M. Ohno, T. Shimokawabe, and T. Aoki, IOP Conf. Ser.: Mater. Sci. Eng. (in press).

39. S. Chen and G.D. Doolen, Annu. Rev. Fluid Mech. 30, 329 (1998).

40. X. Shan and H. Chen, Phys. Rev. E 47, 1815 (1993).

41. J.J. Hoyt, B. Sadigh, M. Asta, and S.M. Foiles, Acta Mater. 47, 3181 (1999).

42. M. Asta, J.J. Hoyt, and A. Karma, Phys. Rev. B 66, 100101 (2002).

43. J.R. Morris, Phys. Rev. B 66, 144104 (2002).

44. J.J. Hoyt, M. Asta, and A. Karma, Mater. Sci. Eng. $R$ 41, 121 (2003).

45. D.Y. Sun, M. Asta, and J.J. Hoyt, Phys. Rev. B 69, 174103 (2004).

46. Y. Watanabe, Y. Shibuta, and T. Suzuki, ISIJ Int. 50, 1158 (2010).

47. R. Hashimoto, Y. Shibuta, and T. Suzuki, ISIJ Int. 51, 1664 (2011).

48. E. Asadi, M.A. Zaeem, S. Nouranian, and M.I. Baskes, Phys. Rev. B 91, 024105 (2015).

49. E. Asadi, M.A. Zaeem, S. Nouranian, and M.I. Baskes, Acta Mater. 86, 169 (2015).

50. Y. Shibuta, K. Oguchi, and T. Suzuki, ISIJ Int. 52, 2205 (2012).

51. M. Berghoff, M. Selzer, and B. Nestler, Sci. World J. 2013, $564272(2013)$.

52. Y. Shibuta, K. Oguchi, and M. Ohno, Scr. Mater. 86, 20 (2014).

53. F.H. Streitz, J.N. Glosli, and M.V. Patel, Phys. Rev. Lett. 96, 225701 (2006).

54. Y. Shibuta and K. Oguchi, The University of Tokyo, Tokyo, T. Takaki: Kyoto Institute of Technology, Kyoto, and M. Ohno: Hokkaido University, Sapporo, unpublished research (2015).

55. Y. Shibuta, Y. Watanabe, and T. Suzuki, Chem. Phys. Lett. 475, 264 (2009).

56. K. Oguchi, Y. Shibuta, and T. Suzuki, J. Jpn. Inst. Met. 76, $462(2012)$.

57. M.W. Finnis and J.E. Sinclair, Philos. Mag. A 50, 45 (1984).

58. H.J.C. Berendsen, J.P.M. Postma, W.F. van Gunsteren, A. DiNola, and J.R. Haak, J. Chem. Phys. 81, 3684 (1984).

59. H.C. Andersen, J. Chem. Phys. 72, 2384 (1980).

60. Y. Shibuta, S. Takamoto, and T. Suzuki, ISIJ Int. 48, 1582 (2008). 
61. Y. Shibuta and T. Suzuki, Chem. Phys. Lett. 445, 265 (2007).

62. Y. Shibuta and T. Suzuki, J. Chem. Phys. 129, 144102 (2008).

63. Y. Qi, T. Çağin, W.L. Johnson, and W.A. Goddard III, J. Chem. Phys. 115, 385 (2001).

64. F. Ding, A. Rosén, S. Curtarolo, and K. Bolton, Appl. Phys. Lett. 88, 133110 (2006).

65. Y. Shibuta and T. Suzuki, Phys. Chem. Chem. Phys. 12, 731 (2010).

66. Y. Shibuta and T. Suzuki, Chem. Phys. Lett. 502, 82 (2011).

67. T. Li, D. Donadio, L.M. Ghiringhelli, and G. Galli, Nat. Mater. 8, 726 (2009).

68. M. Greenwood, M. Haataja, and N. Provatas, Phys. Rev. Lett. 93, 246101 (2004).

69. C.W. Lan and C.J. Shih, Phys. Rev. E 69, 031601 (2004).

70. J.C. Ramirez and C. Beckermann, Acta Mater. 53, 1721 (2005).

71. H. Emmerich and R. Siquieri, J. Phys.: Condens. Matter. 18, 11121 (2006).

72. A. Parisi and M. Plapp, Acta Mater. 56, 1348 (2008).

73. J. Rosam, P.K. Jimack, and A.M. Mullis, Acta Mater. 56, 4559 (2008).

74. J. Rosam, P.K. Jimack, and A.M. Mullis, Phys. Rev. E 79, 030601 (2009).

75. K. Oguchi and T. Suzuki, ISIJ Int. 49, 1536 (2009).

76. M. Ohno and K. Matsuura, Acta Mater. 58, 6134 (2010).

77. M. Ohno and K. Matsuura, ISIJ Int. 50, 1879 (2010).

78. G. Boussinot, E.A. Brener, and D.E. Temkin, Acta Mater. 58,1750 (2010).

79. B. Echebarria, A. Karma, and S. Gurevich, Phys. Rev. E 81, 021608 (2010).

80. S. Gurevich, A. Karma, M. Plapp, and R. Trivedi, Phys. Rev. E 81, 011603 (2010).

81. Z. Wang, J. Wang, J. Li, G. Yang, and Y. Zhou, Phys. Rev. E 84, 041604 (2011).

82. C.A. Gandin, T. Carozzani, H. Digonnet, S. Chen, and G. Guillemot, JOM 65, 1122 (2013).

83. M. Eshraghi, S.D. Felicelli, and B. Jelinek, J. Cryst. Growth 354, 129 (2012).
84. B. Jelinek, M. Eshraghi, S. Felicelli, and J.F. Peters, Comput. Phys. Commun. 185, 939 (2014).

85. N. Provatas, N. Goldenfeld, and J. Dantzig, Phys. Rev. Lett. 80, 3308 (1998).

86. N. Provatas, N. Goldenfeld, and J. Dantzig, J. Comput. Phys. 148, 265 (1999).

87. C.W. Lan, Y.C. Chang, and C.J. Shih, Acta Mater. 51, 1857 (2003).

88. T. Takaki, T. Fukuoka, and Y. Tomita, J. Cryst. Growth 283, 263 (2005).

89. Z. Guo and S.M. Xiong, Comput. Phys. Commun. 190, 89 (2015).

90. D. Tourret and A. Karma, Acta Mater. 82, 64 (2015).

91. A. Yamanaka, T. Aoki, S. Ogawa, and T. Takaki, J. Cryst. Growth 318, 40 (2011).

92. T. Shimokawabe, T. Aoki, T. Takaki, A. Yamanaka, A. Nukada, T. Endo, N. Maruyama, and S. Matsuoka, Proceedings of 2011 SC-International Conference for High Performance Computing, Networking, Storage and Analysis, 1 (2011).

93. D. Walton and B. Chalmers, Trans. Metall. Soc. AIME 215 , 447 (1959)

94. H. Esaka, M. Tamura, and K. Shinozuka, Mater. Trans. 44 , 829 (2003)

95. N. D’Souza, M.G. Ardakani, A. Wagner, B.A. Shollock, and M. McLean, J. Mater. Sci. 37, 481 (2002).

96. Y.Z. Zhou, A. Volek, and N.R. Green, Acta Mater. 56, 2631 (2008).

97. Z. Liu, M. Lin, D. Yu, X. Zhou, Y. Gu, and H. Fu, Metall. Mater. Trans. A 44, 5113 (2013).

98. C. Yang, L. Liu, X. Zhao, N. Wang, J. Zhang, and H. Fu, J. Alloys Compd. 578, 577 (2013).

99. S. Akamatsu and T. Ihle, Phys. Rev. E 56, 4479 (1997).

100. A. Pocheau, J. Deschamps, and M. Georgelin, JOM 59-57, 71 (2007).

101. J. Deschamps, M. Georgelin, and A. Pocheau, Phys. Rev. E 78, 011605 (2008).

102. A. Pocheau, J. Deschamps, and M. Georgelin, Phys. Rev. E 81,051608 (2010) 